\title{
El anegamiento afecta el crecimiento y producción de biomasa en tomate chonto (Solanum lycopersicum L.), cultivado bajo sombrío
}

\section{Waterlogging affects the growth and biomass production of chonto tomatoes (Solanum lycopersicum L.), cultivated under shading}

\section{ADRIANA BARACALDO' \\ RUBÉN CARVAJAL ${ }^{1}$ \\ ÁNGELA PATRICIA ROMERO² \\ ANDREA MARCELA PRIETO² \\ FRANCISCO JAVIER GARCÍA ${ }^{2}$ \\ GERHARD FISCHER ${ }^{3,4}$ \\ DIEGO MIRANDA ${ }^{3}$}

Tomate chonto, híbrido larga vida Roble F1, en fructificación.

Foto: Impulsemillas, Bogotá.

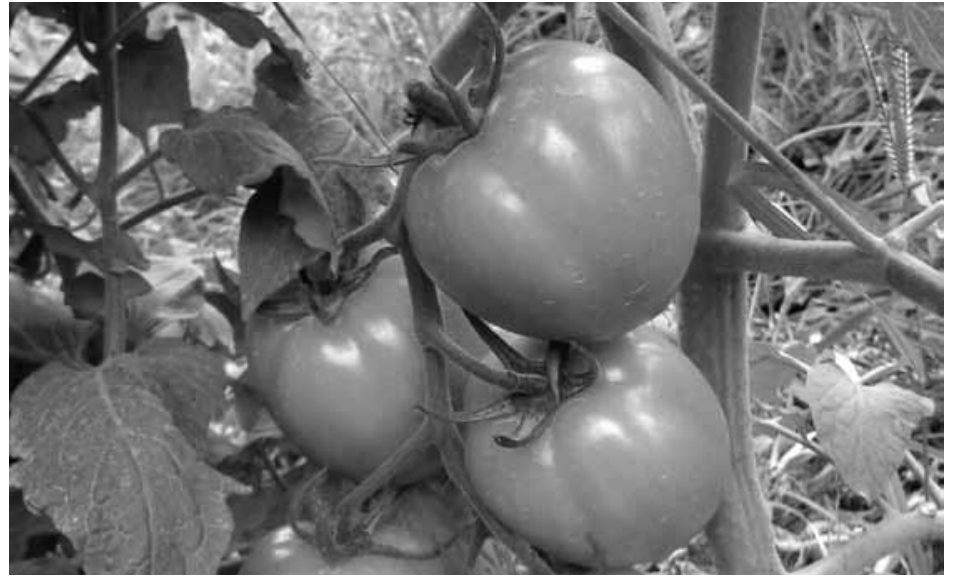

\section{RESUMEN}

El estrés por anegamiento e inundación de las plantaciones se ha convertido en un factor abiótico limitante del crecimiento, el desarrollo y la productividad de los cultivos. Para estudiar el efecto del anegamiento sobre el tomate tipo chonto, híbrido larga vida Roble F1, se realizó un experimento en condiciones de invernadero de plástico, con y sin sombrío (una polisombra negra con 56,4\% de reducción lumínica en un día soleado) de plantas anegadas. Se utilizaron materas plásticas de $1 \mathrm{~L}$ de capacidad, con turba rubia como sustrato en mezcla con suelo orgánico en proporción 1:1, las cuales fueron anegadas con una lámina de agua hasta $4 \mathrm{~cm}$ por encima del sustrato durante 4, 8 y 12 días. Las variables evaluadas incluyeron altura de planta, número de hojas y frutos, y biomasa seca de los órganos. El estudio mostró que los periodos de 4, 8 y 12 días de anegamiento redujeron significativamente la altura de la planta y el número de hojas, después de 12 y 16 días de iniciado el anegamiento, respectivamente, mientras el número de frutos en las plantas anegadas fue significativamente menor a los 40 dda cuando terminó la evaluación. La hipoxia radical en las plantas anegadas durante 8 y 12 días, redujo la producción de biomasa seca, especialmente en frutos y raíces, mientras la interacción 12-días-anegamiento y sombrío disminuyó la masa seca total de la planta. La planta de tomate, en general, parece estar adaptada al efecto de este tipo de sombrío. Con el aumento de la duración del anegamiento aparecieron síntomas como clorosis y caída de hojas basales, además de la epinastia y coloración roja foliar, así mismo la formación de raíces adventicias.

Facultad de Ciencias Agrarias, Programa de Maestría en Ciencias Agrarias, Universidad Nacional de Colombia (Bogotá).

Facultad de Ciencias Agrarias, Programa de Ingeniería Agronómica, Universidad Nacional de Colombia (Bogotá).

Facultad de Ciencias Agrarias, Departamento de Agronomía, Universidad Nacional de Colombia (Bogotá).

Autor para correspondencia.gfischer@unal.edu.co 
Palabras clave adicionales: altura planta, número hojas, masa seca, sintomatología, epinastia, raíces adventicias.

\section{ABSTRACT}

Waterlogging and flooding stress have become major abiotic stress factors for the growth, development and productivity of crops. To study the effect of waterlogging on the chonto tomato long-life Roble F1 hybrid, an experiment was conducted in a plastic greenhouse, with and without black shade netting (with the $56.4 \%$ light reduction on a sunny day). Plastic pots (1 L capacity) were used with blond peat mixed with organic soil at a 1:1 proportion, which were waterlogged up to $4 \mathrm{~cm}$ above the substrate for 4,8 and 12 days. The assessed variables included plant height, number of leaves and fruits, and dry biomass of the organs. The study showed that 4, 8, and 12 days of waterlogging reduced the plant height and number of leaves after 12 and 16 days from waterlogging initiation, respectively; while the number of fruits was significantly lower at 40 dwi, when the evaluation period finished. The root hypoxia in the plants waterlogged for 8 and 12 days reduced plant dry biomass production, especially in the fruits and roots; while the 12-days-waterlogging x shading interaction impaired the total plant dry mass. In general, the tomato plant seems to be adapted to the effect of this type of shading. Increased periods of waterlogging were accompanied by symptoms such as chlorosis, abscission of basal leaves, leaf epinasty, reddish coloring, and formation of adventitious roots.

Additional key words: plant height, leaf number, dry mass, symptomatology, epinasty, adventitious roots.
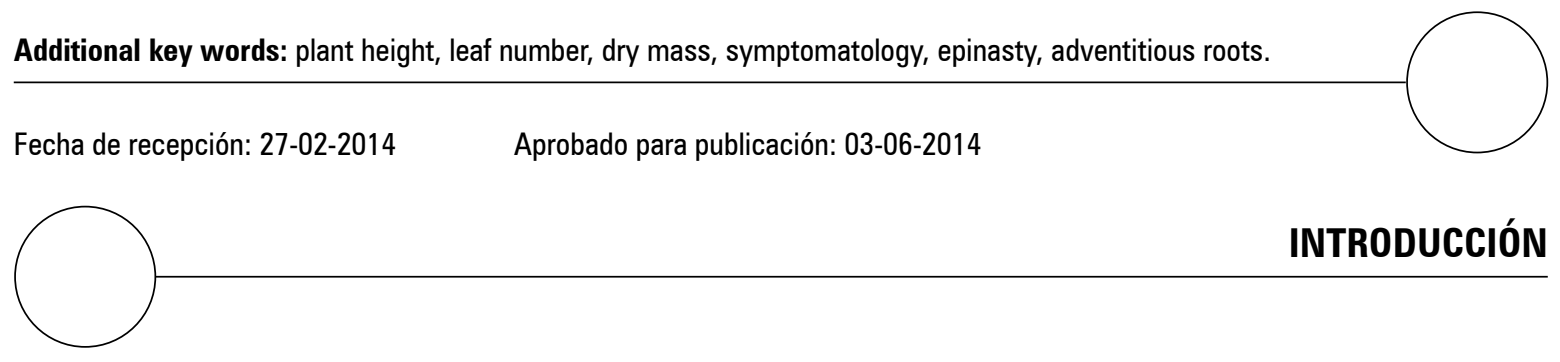

INTRODUCCIÓN

El tomate (Solanum lycopersicum L.) es una de las hortalizas de mayor importancia en el mundo, por su área sembrada y su alto nivel de consumo que alcanza el segundo lugar, después de la papa (Nicola et al., 2009). En Colombia, el cultivo de tomate está disperso por todo el país, cultivándose en 18 departamentos; sin embargo, cerca del 80\% de la producción está concentrada en los departamentos de Cundinamarca, Norte de Santander, Valle del Cauca, Caldas, Huila Risaralda y Antioquia, donde tradicionalmente se siembran tomates tipo chonto y milano (Corpoica, 2006).

Debido al cambio climático, la adversidad en las plantaciones por anegamiento e inundación se ha convertido en un importante factor de estrés abiótico que afecta el crecimiento, desarrollo, la productividad y la distribución de las especies (Jackson y Colmer, 2005). En América Latina, alrededor de $11,3 \%$ de las tierras cultivables presentan drenajes pobres, principalmente porque la fisiografía promueve la inundación, los niveles freáticos altos o las aguas superficiales estancadas (Wood et al., 2000). El anegamiento es un factor limitante en la producción de varios cultivos importantes en Colombia, como por ejemplo la uchuva (Physalis peruviana L.) que disminuye su crecimiento y producción marcadamente a partir de 6 días de anegadas (Aldana et al., 2014).

En tomate, el anegamiento redujo el crecimiento del tallo, área foliar, cuajamiento y rendimiento de los frutos (López y Del Rosario, 1983). Sin embargo, el tomate está clasificado como susceptible a condiciones de anegamiento (Ezin et al., 2010; Rao y Li, 2003), responde al anegamiento con la formación de hojas epinásticas y raíces adventicias que alivian este tipo de estrés (Sauter, 2013). 
La intensidad lumínica la caracteriza Ulrich (1997) como el factor más importante en la ecofisiología de las plantas, ya que durante el día y a lo largo del año presenta una gran variabilidad disminuyendo mucho durante la época pluviosa (Fischer y Pérez, 2012). Con el aumento de la radiación lumínica incrementa el consumo de agua y nutrientes en el tomate, hasta llegar a un nivel máximo de saturación (Canovas, 1995). Para el tomate, la luz interceptada es un factor determinante para el crecimiento y la producción de biomasa y depende mucho del área foliar de la planta (Heuvelink y Dorais, 2005).

El efecto del cambio climático en Colombia, con inundaciones de grandes áreas en el país, no ha sido investigado en el cultivo de tomate, por lo cual el objetivo de este estudio fue analizar el crecimiento y la posible recuperación de tomate, tipo chonto, híbrido larga vida Roble F1, sometidos a estrés por anegamiento en tres intervalos de tiempo y bajo dos condiciones de disponibilidad de luz.

\section{MATERIALES Y MÉTODOS}

El estudio se llevó a cabo en el invernadero de Facultad de Ciencias Agrarias, Universidad Nacional de Colombia, sede Bogotá, localizado a 2.556 $\mathrm{msnm}$, con una temperatura interna promedio de $17^{\circ} \mathrm{C}$ y $80 \%$ de humedad relativa.

Se usaron plantas de tomate tipo chonto (Solanum lycopersicum L.), híbrido larga vida Roble F1, obtenidas de viveros de la empresa Impulsemillas Ltda., Bogotá. El transplante se realizó el día 18 de octubre de 2013, a los 30 días después de la siembra (dds), colocando una plántula por matera de plástico (volumen de $1 \mathrm{~L}, 23 \times 18 \mathrm{~cm}$ ) utilizando como sustrato una mezcla de turba rubia y suelo orgánico (pH 5,78; CO 4,47\%; N 0,39\%; Ca 2,41, Mg 0,16, K 0,05 meq/100 g; P 0,06, Mn 60, Zn 38, Fe 188, Cu 22,3 y B 24,1 mg kg-1) en una relación 1:1, manteniendo una lámina de $4 \mathrm{~cm}$ de agua sobre la superficie del sustrato. El análisis de agua de riego mostró los siguientes valores: $\mathrm{Cl} 2, \mathrm{SO}_{4} 82, \mathrm{PO}_{4} 0,39, \mathrm{NO}_{3}$ 23,4, Ca 39,8, Na 14,8 y $\mathrm{NH}_{4} 0,38 \mathrm{mg} \mathrm{L}^{-1}$.

En las plantas juveniles, con una edad de $60 \mathrm{~d}$ después de la siembra y una altura entre 33 y 36 $\mathrm{cm}$ (tabla 1) y para confirmar resultados con otras variedades de tomate en ese estado de crecimiento reportados por Rao y Li (2003), el anegamiento se inició el 18 de noviembre de 2013, sin incluir el testigo. Según el tiempo de duración se establecieron los tratamientos $0,4,8$ y $12 \mathrm{~d}$ de anegamiento. Al mismo tiempo estos tratamientos se evaluaron bajo una radiación del $100 \%$ por debajo del plástico del invernadero y bajo una polisombra, en malla de polietileno de $50 \%$ de densidad, color negro que transmitió el 43,6\% de la radiación en un día soleado (promedio $2.202,3 \mu \mathrm{mol} \mathrm{m} \mathrm{m}^{-2} \mathrm{~s}^{-1}$ ) y el $37,7 \%$ en un día nublado $\left(482,5 \mu \mathrm{mol} \mathrm{m} \mathrm{m}^{-2} \mathrm{~s}^{-1}\right)$.

Las plantas se fertilizaron semanalmente, iniciando $15 \mathrm{~d}$ después del trasplante con un fertilizante en una relación de $10 \mathrm{~N}-40 \mathrm{P}_{2} \mathrm{O}_{5}-10 \mathrm{~K}_{2} \mathrm{O}$ - $7 \mathrm{~S}$ 0,35 Zn en una solución de 0,5\%. Luego se aplicó dos veces por semana $0,5 \%$ del fertilizante crecimiento (relación $41 \mathrm{~N}-3 \mathrm{P}_{2} \mathrm{O}_{5}-3 \mathrm{~K}_{2} \mathrm{O}-0,06 \mathrm{CaO}$ $0,2 \mathrm{MgO}-0,5 \mathrm{~S}-0,04 \mathrm{~B}-0,03 \mathrm{Cu}-0,03 \mathrm{Fe}-0,1 \mathrm{Mn}$ - 0,005 Mo - 0,1 Zn). Cuando las plantas iniciaron su estado de prefloración se aplicó dos veces por semana $0,5 \%$ del fertilizante producción (relación 10 $\mathrm{N}-3 \mathrm{P}_{2} \mathrm{O}_{5}-40 \mathrm{~K}_{2} \mathrm{O}-1 \mathrm{MgO}-1 \mathrm{~S}-0,2 \mathrm{~B}-0,03$ $\mathrm{Cu}$ ). Para el control fitosanitario se aplicaron productos como oxicloruro de cobre, mancozeb, metalaxyl, trifloxystrobin, tebuconazole y clorpirifos.

La toma de datos se realizó cada 4 d, hasta los 20 días de iniciado el anegamiento (ddia) y al final del estudio, a los 40 ddia, en los que se midió la longitud del tallo principal, el número de botones florales y flores, el número de frutos, el número de hojas activas y hojas secas y la cantidad de biomasa seca de los órganos de la planta. Se utilizó una balanza electrónica $(0,001$ g de precisión; Precisa Adventurer, OHAUS, Parsippany, NJ) para peso seco de hojas, tallos, flores y frutos y raíces, después de haber sido sometidas a secado en una estufa a $75^{\circ} \mathrm{C}$ por $72 \mathrm{~h}$. 
Se utilizó un diseño experimental completamente al azar con ocho tratamientos y cuatro repeticiones, en un arreglo de parcelas subdivididas donde la parcela principal fue el sombreamiento. La unidad experimental consistió en nueve plantas para un total de 288 plantas. El análisis de datos se realizó con el paquete estadístico SAS V 9.2 en el cual se determinó el análisis de varianza (Anova). El parámetro de crecimiento de la biomasa seca total determinó empleando el enfoque funcional del modelo Gompertz y las diferencias estadísticas de las medias se compararon por el método de Tukey-Kramer $(P \leq 0,05)$.

\section{RESULTADOS Y DISCUSIÓN}

\section{Altura de la planta}

Como aparece en la tabla 1 , no se observan diferencias significativas $(P>0,05)$ en la altura de las plantas a los 4 y 8 ddia, sin embargo, a los 12 ddia en los tratamientos con plantas anegadas estas tuvieron tallos más cortos $(P \leq 0,05)$ frente al testigo, y estas diferencias aumentaron hasta el día 40 de la observación. En general, no hubo diferencias estadísticas entre las plantas sin y con polisombra, solamente como interacción, las plantas anegadas durante $8 \mathrm{~d}$ y con sombra mostraron reducciones significativas $(P \leq 0,05)$ a los 12 ddia, comparadas con el testigo y a los 16 ddia de las anegadas sin sombra, indicando que el estrés por anegamiento fue intensificado por la reducción de luz. No hubo una clara tendencia si el aumento del estrés de 4 a $12 \mathrm{~d}$ de anegamiento afectó diferencialmente la altura de la planta, sino ya $4 \mathrm{~d}$ fueron suficientes para disminuir el crecimiento del tallo como lo encontraron Kuo y Chen (1980) en plantas de tomate de 1 mes de edad y anegadas durante $3 \mathrm{~d}$.

La disminución del crecimiento del tallo en plantas de tomate anegadas, a partir de $4 \mathrm{~d}$, coincide con lo observado por Aldana et al. (2014) en uchuva sometida a tratamientos similares de anegamiento. Estos autores, adhiriéndose a los resultados de Bradford y Yang (1981), atribuyeron esta reducción a un menor suministro de citoquininas y giberelinas, sintetizadas en las raíces estresadas hacia el tallo. Además, la drástica reducción del crecimiento longitudinal en 8 y 12 $\mathrm{d}$ de anegamiento puede ser también influencia-

Tabla 1. Promedio de la longitud de tallo $(\mathrm{cm})$ de plantas de tomate cultivadas con y sin polisombra y sometidas a anegamiento durante 0 (testigo), 4, 8 y 12 días, evaluadas durante 40 días.

\begin{tabular}{|c|c|c|c|c|c|c|c|}
\hline \multirow{2}{*}{ Tratamientos } & \multicolumn{7}{|c|}{ Días } \\
\hline & 0 & 4 & 8 & 12 & 16 & 20 & 40 \\
\hline $\begin{array}{l}\text { Sin sombra, sin aneg. } \\
\text { Con sombra, sin aneg. } \\
\text { Sin sombra, aneg. } 4 \mathrm{~d} \\
\text { Con sombra, aneg. } 4 \mathrm{~d} \\
\text { Sin sombra, aneg. } 8 \mathrm{~d} \\
\text { Con sombra, aneg. } 8 \mathrm{~d} \\
\text { Sin sombra, aneg. } 12 \mathrm{~d} \\
\text { Con sombra, aneg. } 12 \mathrm{~d}\end{array}$ & $\begin{array}{l}34,75 a \\
32,75 a \\
34,75 a \\
34,50 a \\
34,50 a \\
36,25 a \\
34,25 a \\
32,75 a\end{array}$ & $\begin{array}{l}39,00 \mathrm{a} \\
42,50 \mathrm{a} \\
37,65 \mathrm{a} \\
39,73 \mathrm{a} \\
37,67 \mathrm{a} \\
37,73 \mathrm{a} \\
37,67 \mathrm{a} \\
38,73 \mathrm{a}\end{array}$ & $\begin{array}{l}44,30 \mathrm{a} \\
45,20 \mathrm{a} \\
39,50 \mathrm{a} \\
40,45 \mathrm{a} \\
38,80 \mathrm{a} \\
38,16 \mathrm{a} \\
38,40 \mathrm{a} \\
39,78 \mathrm{a}\end{array}$ & $\begin{array}{l}49,13 a \\
46,88 a b \\
39,38 a b \\
40,75 a b \\
40,05 a b \\
38,88 \mathrm{~b} \\
38,88 \mathrm{~b} \\
40,63 \mathrm{ab}\end{array}$ & $\begin{array}{l}53,50 \mathrm{a} \\
55,75 \mathrm{a} \\
40,75 \mathrm{bc} \\
47,00 \mathrm{~b} \\
41,00 \mathrm{bc} \\
39,00 \mathrm{~d} \\
40,50 \mathrm{~cd} \\
41,50 \mathrm{bc}\end{array}$ & $\begin{array}{l}56,00 \mathrm{a} \\
57,50 \mathrm{a} \\
41,75 \mathrm{~b} \\
43,05 \mathrm{ab} \\
42,50 \mathrm{~b} \\
40,50 \mathrm{~b} \\
41,25 \mathrm{~b} \\
42,25 \mathrm{~b}\end{array}$ & $\begin{array}{l}59,50 a \\
58,75 a \\
43,25 b \\
46,25 a b \\
43,50 b \\
42,00 b \\
40,00 b \\
43,50 b\end{array}$ \\
\hline $\begin{array}{l}\text { Sin polisombra } \\
\text { Con polisombra }\end{array}$ & $\begin{array}{l}34,56 \text { a } \\
34,06 \text { a }\end{array}$ & $\begin{array}{l}38,00 \mathrm{a} \\
39,68 \mathrm{a}\end{array}$ & $\begin{array}{l}40,25 a \\
40,90 a\end{array}$ & $\begin{array}{l}41,86 a \\
41,75 a\end{array}$ & $\begin{array}{l}44,19 \mathrm{a} \\
44,56 \mathrm{~b}\end{array}$ & $\begin{array}{l}44,94 \mathrm{a} \\
46,88 \mathrm{a}\end{array}$ & $\begin{array}{l}46,56 \text { a } \\
47,63 \text { a }\end{array}$ \\
\hline $\begin{array}{l}\text { Anegamiento } 0 \mathrm{~d} \\
\text { Anegamiento } 4 \mathrm{~d} \\
\text { Anegamiento } 8 \mathrm{~d} \\
\text { Anegamiento } 12 \mathrm{~d}\end{array}$ & $\begin{array}{l}33,75 a \\
34,62 a \\
35,37 a \\
33,50 a\end{array}$ & $\begin{array}{l}40,75 a \\
38,69 a \\
37,70 a \\
38,20 a\end{array}$ & $\begin{array}{l}44,75 a \\
39,98 a \\
38,48 a \\
39,09 a\end{array}$ & $\begin{array}{l}48,00 a \\
40,07 b \\
39,69 b \\
39,76 b\end{array}$ & $\begin{array}{l}54,63 a \\
43,87 b \\
40,00 b \\
41,00 b\end{array}$ & $\begin{array}{l}56,75 a \\
42,40 b \\
41,50 b \\
41,75 b\end{array}$ & $\begin{array}{l}59,12 a \\
44,75 b \\
42,75 b \\
41,75 b\end{array}$ \\
\hline
\end{tabular}

Promedios en la misma columna con letras distintas indican diferencia significativa según la prueba de Tukey $(P \leq 0,05)$. 
dos por el etileno (Shiu et al., 1998) cuya síntesis es mayor bajo condiciones de anoxia debido a la mayor producción de la aminociclopropano carboxilasa (ACC) en las raíces y su transporte al tallo y, en las hojas, es convertido por ACC oxidasa a etileno (Lorbieke y Sauter, 1999).

Por otro lado, Jordán y Casaretto (2006) atribuyeron este efecto a una acción inhibidora del ácido abscísico (ABA), el cual se expresa mejor en condiciones de falta de oxígeno; ya sea por exceso o déficit hídrico, ABA es capaz de inhibir la elongación de tallos más que de raíces y postulan que el mecanismo por el cual ABA inhibe el crecimiento de tallos es a través de un bloqueo de bombas de protones activada por auxinas, notándose una disminución de la acidificación de la pared celular y con ello en la elongación celular.

\section{Número de hojas}

Como muestra la tabla 2 , el número de hojas disminuyó significativamente $(P \leq 0,05)$ en las plantas con los tratamientos de anegamiento comparadas con el testigo, a partir de 12 ddia en plantas anegadas durante 8 y $12 \mathrm{~d}$ y a partir de 16 ddia en las estresadas durante $4 \mathrm{~d}$. Además, el anegamiento causó una mayor cantidad de hojas muertas que las plantas testigo a los $20 \mathrm{y}$ 40 ddia (tabla 2). Este resultado coincide con lo observado por Aldana et al. (2014) en uchuvas, anegadas durante 6 y $8 \mathrm{~d}$, que tuvieron menos de la mitad de las hojas que las plantas anegadas durante 0,2 y $4 \mathrm{~d}$.

El menor número de hojas en plantas anegadas se debe, por un lado, al menor crecimiento de las plantas estresadas como confirma la reducida longitud del tallo (tabla 1) y la mayor abscisión de las hojas bajeras como describen Das (2012) y Kozlowski y Pallardy (1997) para este tipo de estrés.

La reducción del área foliar puede ser interpretada como una eficiencia en la redistribución de los fotoasimilados y en un ahorro energético, cuando los procesos fotosintéticos son menos eficientes y las pérdidas por mantenimiento son más altas que la energía producida (Reich y Walters, 1992).

Tabla 2. Promedio del número de hojas de plantas de tomate cultivadas con y sin polisombra y sometidas a anegamiento durante 0 (testigo), 4, 8 y 12 días, indicando también las hojas vivas y muertas a 20 y 40 días.

\begin{tabular}{|c|c|c|c|c|c|c|c|c|c|}
\hline \multirow[b]{3}{*}{ Tratamientos } & \multicolumn{9}{|c|}{ Días } \\
\hline & \multirow[b]{2}{*}{0} & \multirow[b]{2}{*}{4} & \multirow[b]{2}{*}{8} & \multirow[b]{2}{*}{12} & \multirow[b]{2}{*}{16} & \multicolumn{2}{|c|}{20} & \multicolumn{2}{|c|}{40} \\
\hline & & & & & & Hojas vivas & $\begin{array}{l}\text { Hojas } \\
\text { muertas }\end{array}$ & Hojas vivas & $\begin{array}{l}\text { Hojas } \\
\text { muertas }\end{array}$ \\
\hline Sin sombra, sin aneg. & $10,25 a$ & $10,75 a$ & $11,25 a$ & $12,25 \mathrm{a}$ & $12,50 \mathrm{a}$ & $14,75 \mathrm{a}$ & $0,50 \mathrm{c}$ & $14,25 \mathrm{a}$ & $2,75 b$ \\
\hline Con sombra, sin aneg. & $10,25 a$ & $11,00 \mathrm{a}$ & $11,25 a$ & $12,00 \mathrm{a}$ & $12,50 \mathrm{a}$ & $12,75 \mathrm{ab}$ & $1,25 b$ & $13,75 \mathrm{a}$ & $2,50 \mathrm{~b}$ \\
\hline Sin sombra, aneg. $4 \mathrm{~d}$ & $10,50 \mathrm{a}$ & $11,00 \mathrm{a}$ & $11,25 a$ & $11,25 \mathrm{a}$ & $11,75 a b$ & $12,25 b$ & $2,75 a b$ & $13,25 \mathrm{a}$ & $3,50 a b$ \\
\hline Con sombra, aneg. $4 \mathrm{~d}$ & $10,25 a$ & $11,00 \mathrm{a}$ & $11,25 a$ & $11,50 \mathrm{a}$ & $11,50 \mathrm{ab}$ & $12,25 \mathrm{~b}$ & $3,50 \mathrm{a}$ & $12,00 \mathrm{~b}$ & $3,25 a b$ \\
\hline Sin sombra, aneg. $8 \mathrm{~d}$ & $9,75 a$ & $10,50 \mathrm{a}$ & $10,75 a$ & $11,00 a b$ & $11,00 \mathrm{~b}$ & $12,50 a b$ & $1,50 \mathrm{~b}$ & $13,50 \mathrm{a}$ & $3,25 a b$ \\
\hline Con sombra, aneg. $8 \mathrm{~d}$ & $10,25 a$ & $10,75 a$ & $10,75 a$ & $10,50 \mathrm{a}$ & $10,75 b$ & $12,25 b$ & $1,25 b$ & $12,00 \mathrm{ab}$ & $3,75 a$ \\
\hline Sin sombra, aneg. $12 \mathrm{~d}$ & $10,50 \mathrm{a}$ & $11,00 \mathrm{a}$ & $10,25 a$ & $9,75 b$ & $10,25 \mathrm{bc}$ & $10,38 \mathrm{bc}$ & $3,50 \mathrm{a}$ & $10,75 b$ & $4,25 \mathrm{a}$ \\
\hline Con sombra, aneg. $12 \mathrm{~d}$ & $10,00 \mathrm{a}$ & $10,75 a$ & $10,00 \mathrm{a}$ & $9,75 b$ & $10,00 \mathrm{c}$ & $11,00 \mathrm{c}$ & $3,50 \mathrm{a}$ & $10,75 b$ & $5,25 \mathrm{a}$ \\
\hline Sin polisombra & $10,31 \mathrm{a}$ & $10,81 \mathrm{a}$ & $10,88 a$ & $11,06 \mathrm{a}$ & $11,37 \mathrm{a}$ & $12,47 \mathrm{a}$ & $2,06 \mathrm{a}$ & $12,94 \mathrm{a}$ & $3,43 \mathrm{a}$ \\
\hline Con polisombra & $10,12 a$ & $10,88 \mathrm{a}$ & $10,81 \mathrm{a}$ & $10,94 \mathrm{a}$ & $11,18 \mathrm{a}$ & $12,06 \mathrm{a}$ & $2,37 \mathrm{a}$ & $12,13 \mathrm{a}$ & 3,69 a \\
\hline Anegamiento $0 \mathrm{~d}$ & $10,00 \mathrm{a}$ & $10,88 \mathrm{a}$ & $11,25 a$ & $12,13 \mathrm{a}$ & $12,50 \mathrm{a}$ & $13,75 a$ & $1,00 \mathrm{~b}$ & $14,00 \mathrm{a}$ & $2,63 \mathrm{~b}$ \\
\hline Anegamiento $4 \mathrm{~d}$ & $10,25 a$ & $11,00 \mathrm{a}$ & $11,25 a$ & $11,38 \mathrm{ab}$ & $11,63 \mathrm{~b}$ & $12,25 b$ & $1,25 \mathrm{~b}$ & $12,63 \mathrm{~b}$ & $3,38 a b$ \\
\hline Anegamiento $8 \mathrm{~d}$ & $10,50 \mathrm{a}$ & $10,63 a$ & $10,75 a$ & $10,75 b$ & $10,88 \mathrm{bc}$ & $12,38 b$ & $3,13 a$ & $12,75 b$ & $3,50 \mathrm{a}$ \\
\hline Anegamiento $12 \mathrm{~d}$ & $10,12 a$ & $10,88 \mathrm{a}$ & $10,13 a$ & $9,75 b$ & $10,38 \mathrm{c}$ & $10,69 \mathrm{c}$ & $3,50 \mathrm{a}$ & $10,75 c$ & $4,75 a$ \\
\hline
\end{tabular}

Promedios en la misma columna con letras distintas indican diferencia significativa según la prueba de Tukey $(P \leq 0,05)$. 


\section{Número de frutos}

La tabla 3 muestra que el número de frutos fue afectado por las condiciones de anegamiento, a los 16 ddia, se observó una reducción significativa $(P \leq 0,05)$ en los frutos cuajados en las plantas de $12 \mathrm{~d}$ anegadas, mientras que a los 20 ddia las plantas mostraron indicios de una recuperación del estrés, pero, a los 40 ddia hubo una significativa reducción en el número de los frutos en las plantas anegadas, comparadas con el testigo. En relación con el sombreamiento, no se presentó una clara tendencia, ni diferencia significativa entre este tratamiento principal (tabla 3 ). En cuanto al número de botones florales y flores (datos no mostrados), no se presentaron diferencias significativas entre los tratamientos.

Las condiciones de anegamiento inhiben la formación de botones florales, flores, frutos y su desarrollo en plantas no tolerantes a esta situación de estrés (Kozlowski y Pallardy, 1997). Así Ezin et al. (2010) registraron en tomate una reducción en el número de flores y frutos y su tamaño y peso y atribuyeron esta resultado a la inhibición de la fotosíntesis y otros efectos adversos por la hipoxia que se pudo confirmar en el presente ensayo. Además, los desbalances en la síntesis y el transporte de hormonas, causados por la falta de oxígeno (Bradford y Yang, 1981) alteran los procesos reproductivos que son de alto requerimiento de energía (Fischer et al., 2012).

\section{Masa seca de órganos de la planta}

La variable que fue más afectada, después de la masa seca (MS) de los frutos, fue la MS de las raíces con reducciones significativas $(P \leq 0,05)$, en un $51,7 \%$ y $56,1 \%$ en anegamientos durante 8 y $12 \mathrm{~d}$, respectivamente, comparada con el testigo (tabla 4). Debido a las condiciones hipóxicas la difusión de oxígeno en suelos inundados es muy baja y el intercambio de gases entre la raíz sumergida y el ambiente es muy limitado $\mathrm{y}$, teniendo en cuenta que las plantas como organismos aeróbicos necesitan la disponibilidad de oxígeno para la toma de nutrientes; el agua en exceso alrededor de las raíces puede causar condiciones muy adversas para su crecimiento (Iacona et al., 2012; Kozlowski y Pallardy, 1997). El aire en los poros del suelo es reemplazado por el agua y el oxígeno es consumido rápidamen-

Tabla 3. Promedio del número de frutos de plantas de tomate cultivadas con y sin polisombra y sometidas a anegamiento durante 0 (testigo), 4, 8 y 12 días, evaluadas durante 40 días.

\begin{tabular}{|c|c|c|c|c|}
\hline \multirow{2}{*}{ Tratamientos } & \multicolumn{4}{|c|}{ Días } \\
\hline & 12 & 16 & 20 & 40 \\
\hline $\begin{array}{l}\text { Sin sombra, sin aneg. } \\
\text { Con sombra, sin aneg. } \\
\text { Sin sombra, aneg. } 4 \mathrm{~d} \\
\text { Con sombra, aneg. } 4 \mathrm{~d} \\
\text { Sin sombra, aneg. } 8 \mathrm{~d} \\
\text { Con sombra, aneg. } 8 \mathrm{~d} \\
\text { Sin sombra, aneg. } 12 \mathrm{~d} \\
\text { Con sombra, aneg. } 12 \mathrm{~d}\end{array}$ & $\begin{array}{l}1,0 \mathrm{a} \\
1,0 \mathrm{a} \\
0,7 \mathrm{a} \\
0,8 \mathrm{a} \\
1,5 \mathrm{a} \\
1,5 \mathrm{a} \\
0,5 \mathrm{a} \\
0,3 \mathrm{a}\end{array}$ & $\begin{array}{l}1,0 \mathrm{ab} \\
1,2 \mathrm{a} \\
0,8 \mathrm{bc} \\
0,8 \mathrm{bc} \\
1,8 \mathrm{a} \\
1,5 \mathrm{a} \\
0,7 \mathrm{bc} \\
0,5 \mathrm{c}\end{array}$ & $\begin{array}{l}1,5 \mathrm{a} \\
1,3 \mathrm{a} \\
1,3 \mathrm{a} \\
0,8 \mathrm{a} \\
2,5 \mathrm{a} \\
1,5 \mathrm{a} \\
1,0 \mathrm{a} \\
0,5 \mathrm{ab}\end{array}$ & $\begin{array}{l}4,5 \mathrm{a} \\
2,0 \mathrm{~b} \\
2,5 \mathrm{~b} \\
1,0 \mathrm{~b} \\
2,8 \mathrm{ab} \\
2,3 \mathrm{~b} \\
2,0 \mathrm{~b} \\
1,8 \mathrm{~b}\end{array}$ \\
\hline $\begin{array}{l}\text { Sin polisombra } \\
\text { Con polisombra }\end{array}$ & $\begin{array}{l}0,9 a \\
1,2 a\end{array}$ & $\begin{array}{l}0,9 a \\
1,4 a\end{array}$ & $\begin{array}{l}1,2 \mathrm{a} \\
1,4 \mathrm{a}\end{array}$ & $\begin{array}{l}2,5 a \\
2,2 a\end{array}$ \\
\hline $\begin{array}{l}\text { Anegamiento } 0 \mathrm{~d} \\
\text { Anegamiento } 4 \mathrm{~d} \\
\text { Anegamiento } 8 \mathrm{~d} \\
\text { Anegamiento } 12 \mathrm{~d}\end{array}$ & $\begin{array}{l}1,2 \mathrm{a} \\
1,2 \mathrm{a} \\
1,3 \mathrm{a} \\
0,6 \mathrm{a}\end{array}$ & $\begin{array}{l}1,5 \mathrm{a} \\
1,2 \mathrm{a} \\
1,6 \mathrm{a} \\
0,7 \mathrm{~b}\end{array}$ & $\begin{array}{l}1,5 \mathrm{a} \\
1,2 \mathrm{a} \\
1,8 \mathrm{a} \\
0,7 \mathrm{a}\end{array}$ & $\begin{array}{l}3,6 \mathrm{a} \\
2,1 \mathrm{~b} \\
2,2 \mathrm{~b} \\
1,4 \mathrm{~b}\end{array}$ \\
\hline
\end{tabular}

Promedios en la misma columna con letras distintas indican diferencia significativa según la prueba de Tukey $(P \leq 0,05)$. 
te por la respiración de las raíces y la actividad microbial (Koppitz, 2004) y, consecuentemente, por falta de oxígeno las raíces empiezan a morirse (Armstrong y Drews, 2002).

En situaciones de estrés por anegamiento, el crecimiento de las plantas depende mucho de la efectividad en la captación de recursos, provenientes de la parte aérea, como la subterránea, por lo que la interrupción de la absorción de agua por parte de las raíces puede ocasionar un cierre estomático afectando la fotosíntesis y, en consecuencia, la producción de azúcares en las hojas que, generalmente, se utilizan para el mismo crecimiento de las raíces (Neumann, 1993).

También, la inundación parece reducir la conductividad hidráulica de las raíces y, por otro lado, puede causar un daño directo al sistema radical a causa de la acumulación de productos tóxicos originados por la respiración anaeróbica (Kozlowski y Pallardy, 1997). Mientras que el ácido láctico es el primer producto formado debido a la acidificación citológica (Jiménez et al., 2012), el cambio resultante en el pH de las células favorece rápidamente la formación de acetaldehído, que puede ser muy tóxico, y el cual es convertido en etanol por medio de la actividad de la deshidrogenasa alcohólica (Mittler, 2002). El etanol, producido por la respiración anaeróbica en condiciones de anegamiento puede causar daños importantes, aunque pueden ser contrarrestados con la aplicación de micorrizas (Rutto et al., 2001).
Como respuesta al estrés por inundación se inducen vías de señales de transducción en la planta que llevan al desarrollo de la vía metabólica de la fermentación y posteriormente, de acuerdo con la especie, a la generación de cambios morfológicos y formación de raíces adventicias (Yin et al., 2010). En las plantas de tomate $12 \mathrm{~d}$ anegadas se mostró como resultado la formación de raíces adventicias (figura 1), lo cual confirma estudios realizados por Vidoz et al. (2010) y Perez et al. (1999) al considerar que el tomate es un cultivo conocido por su sensibilidad a la inundación, pero su capacidad de producir raíces adventicias aumenta la supervivencia de la planta cuando disminuye el nivel de oxígeno en la zona radical. También Ezin et al. (2010) observaron en plantas de tomate bajo anegamiento la formación de raíces adventicias, las cuales pueden abastecer la demanda de $\mathrm{O}_{2}$ para su respiración (Parolin, 2009).

La significativamente menor acumulación de MS $(P \leq 0,05)$ en el tallo de plantas anegadas durante 8 y $12 \mathrm{~d}$ coincide con el reducido crecimiento longitudinal (tabla 1) en estos tratamientos. Como se mencionó anteriormente el menor suministro de los fitorreguladores de crecimiento citoquininas y giberelinas por las raíces estresadas al tallo (Bradford y Yang, 1981) y la mayor síntesis de etileno bajo estas condiciones (Shiu et al., 1998) pueden disminuir el crecimiento longitudinal y, así, la acumulación de biomasa.

Siguiendo el patrón de los órganos, también la MS total fue reducida $(P \leq 0,05)$ por el anega-

Tabla 4. Promedio de la masa seca de órganos de plantas de tomate sometidas a anegamiento durante 0 (testigo), 4, 8 y 12 días,evaluada a los 40 días.

\begin{tabular}{|c|c|c|c|c|c|c|}
$\begin{array}{c}\text { Anegamiento } \\
\text { (días) }\end{array}$ & $\begin{array}{c}\text { Raíces } \\
(\mathrm{g})\end{array}$ & $\begin{array}{c}\text { Tallo } \\
(\mathrm{g})\end{array}$ & $\begin{array}{c}\text { Hojas } \\
(\mathrm{g})\end{array}$ & $\begin{array}{c}\text { Flores } \\
(\mathrm{g})\end{array}$ & $\begin{array}{c}\text { Frutos } \\
(\mathrm{g})\end{array}$ & $\begin{array}{c}\text { Total } \\
(\mathrm{g})\end{array}$ \\
\hline 0 & $1,80 \mathrm{a}$ & $2,97 \mathrm{a}$ & $3,76 \mathrm{a}$ & $0,08 \mathrm{a}$ & $21,95 \mathrm{a}$ & $30,56 \mathrm{a}$ \\
\hline 4 & $1,42 \mathrm{ab}$ & $2,00 \mathrm{a}$ & $2,85 \mathrm{ab}$ & $0,07 \mathrm{a}$ & $20,98 \mathrm{a}$ & $27,32 \mathrm{a}$ \\
\hline 8 & $0,87 \mathrm{~b}$ & $1,63 \mathrm{~b}$ & $2,09 \mathrm{~b}$ & $0,05 \mathrm{a}$ & $4,59 \mathrm{~b}$ & $9,24 \mathrm{~b}$ \\
\hline 12 & $0,79 \mathrm{~b}$ & $1,55 \mathrm{~b}$ & $2,11 \mathrm{~b}$ & $0,07 \mathrm{a}$ & $3,52 \mathrm{~b}$ & $8,06 \mathrm{~b}$ \\
\hline
\end{tabular}

Promedios en la misma columna con letras distintas indican diferencia significativa según la prueba de Tukey $(P \leq 0,05)$. 


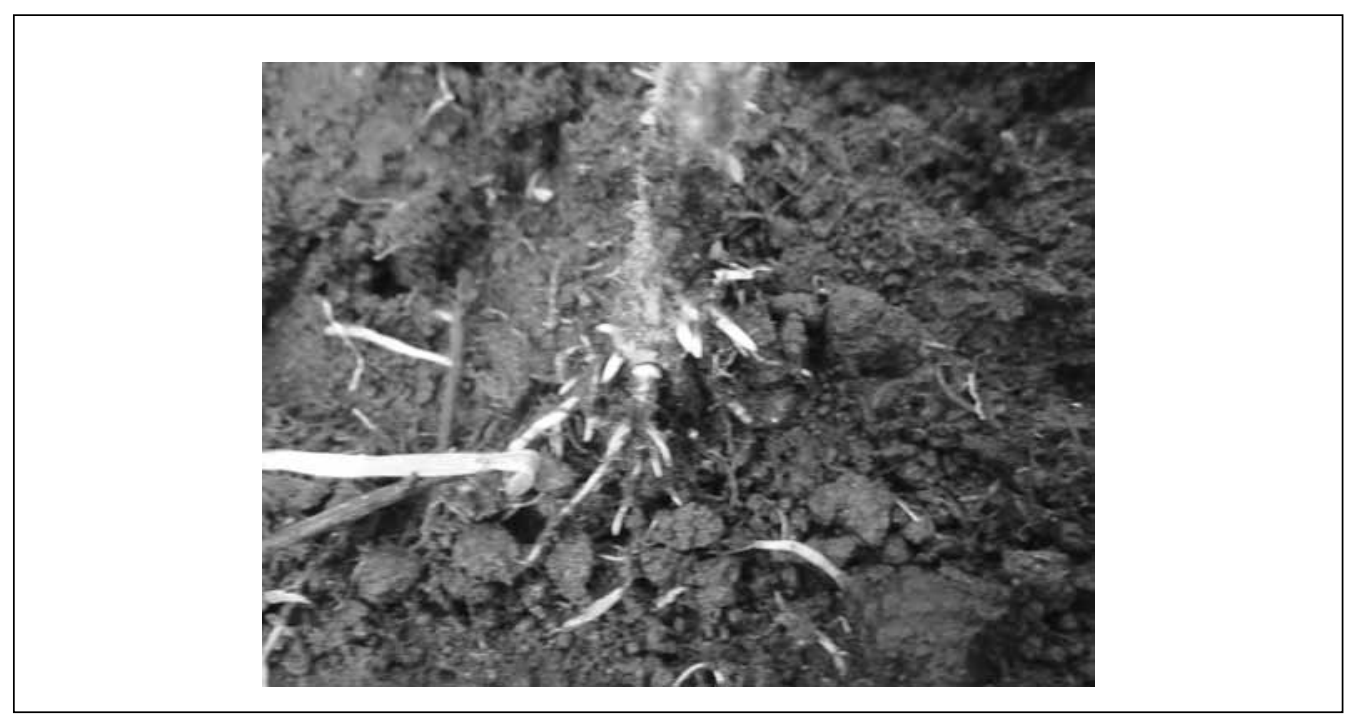

Figura 1. Formación de raíces adventicias en tomates, observadas en plantas de 8 y 12 d anegadas. Foto: R. Carvajal.

miento durante 8 y 12 d (tabla 4) y esta disminución se expresó a partir de los 20 a 24 ddia (figura 2). Empleando un enfoque funcional, se observa en la interacción anegamiento (durante 8 y 12 d x sombra) una disminución en la acumulación de la masa seca de la planta (figura 2). Esta interacción con el factor anegamiento es una de las pocas que se encontró por la colocación de la polisombra sobre el cultivo y confirma lo expresado por Fischer y Orduz-Rodríguez (2012) que la intensidad lumínica deficiente, en ninguna localidad de la producción es un factor tan limitante como sí lo es una temperatura deficiente o el estrés hídrico.

Sin embargo, Mittler (2006) constató que la ocurrencia de una combinación de diferentes tipos de estrés abiótico es única y específica y no se puede extrapolar la respuesta de la planta a cada uno de los dos estreses, aplicados individualmente $y$, por otro lado, las reacciones $y$ aclimataciones de las plantas a estos estreses combinados han sido poco investigadas tanto fisiológica como molecularmente. En este contexto, caben mencionar resultados, obtenidos por Bouzo et al. (2008), quienes evaluaron el efecto del sombrío en cultivos de tomate al aire libre y encontraron que plantas con sombreamiento tuvieron mayor área foliar y peso fresco y seco. Estos autores atribuyeron esta respuesta a que en estos tomates (de crecimiento indeterminado), la reducción de luz sobre las hojas inferiores aceleró el movimiento de nitrógeno a las superiores y, consecuentemente, produjo una mayor concentración de $\mathrm{N}$ y mayor crecimiento en estas hojas más jóvenes.

La menor acumulación de MS total de la planta es un indicio de que en el tomate la capacidad fotosintética es fuertemente inhibida en condiciones de anegamiento (Casierra-Posada y Gómez, 2008) y que el tomate chonto es poco tolerante a la hipoxia radical. En el caso del estrés por anegamiento, la fotosíntesis por efecto de la disminución del potencial hídrico de la planta sufre una drástica caída debido a una reducción de la conductancia estomática, lo cual reduce el abastecimiento y la asimilación del $\mathrm{CO}_{2}$ (Lopez y Kursar, 2003), reduciendo también la capacidad del protoplasma de realizar fotosíntesis debido a su deshidratación. La disminución de la biomasa total de la planta fue reportada también por Aldana et al. (2014) en uchuva, anegada durante 6 y 8 d y por Casierra-Posada y Vargas 


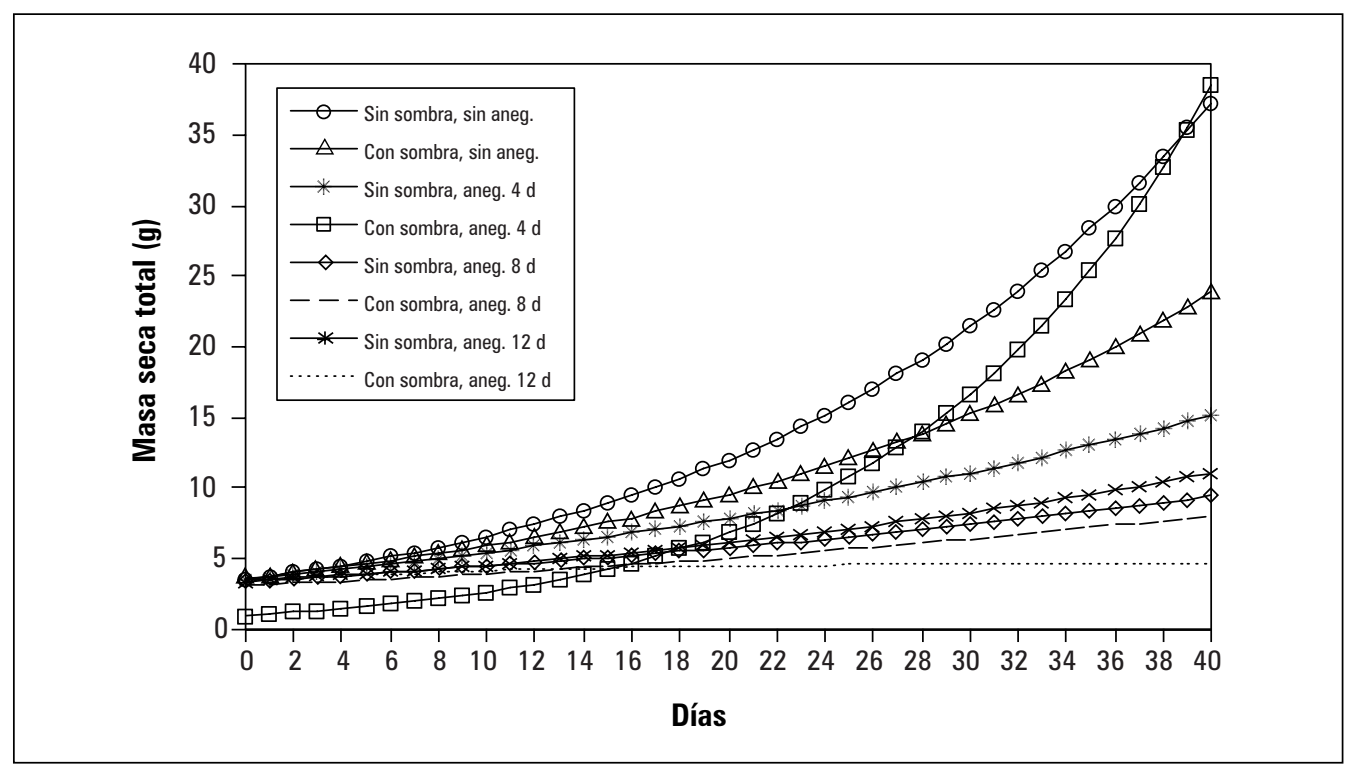

Figura 2. Comportamiento de la masa seca total en plantas de tomate cultivadas con y sin polisombra y sometidas a 0, 4, 8 y 12 días de anegamiento, evaluadas durante 40 días.

(2007) en tres cultivares de fresa encharcados.

Por otro lado, el crecimiento y así la producción de biomasa de la planta es afectada por la disminución de la absorción de nutrientes bajo las condiciones de hipoxia radical, como lo describen Kozlowski y Pallardy (1997) para especies no tolerantes al anegamiento, en las cuales disminuye la absorción de $\mathrm{N}, \mathrm{P}$ y $\mathrm{K}$ en la medida en que la energía se vuelve insuficiente para sostener la absorción de nutrientes imprescindibles para el crecimiento.

\section{Síntomas en las plantas anegadas}

Además de la formación de raíces adventicias, descrita anteriormente, a los 40 ddia las plantas con 4 $\mathrm{d}$ de anegamiento manifestaron pérdida de turgencia en todo el tallo principal, con inicios de senescencia y clorosis en las hojas basales. Las plantas 8 $\mathrm{d}$ anegadas también mostraron hojas poco turgentes, senescencia de hojas basales y enrollamiento de las hojas más jóvenes, mientras las plantas 12 $\mathrm{d}$ anegadas manifestaron pérdida de turgencia, senescencia de hojas basales, con hojas de la mitad del tallo hasta el ápice entorchadas. En todos los tratamientos inundados la consecuencia fue el color rojizo (presencia de antocianinas) en las hojas.

Para Kozlowski y Pallardy (1997) y Rajendran et al. (2012) la clorosis y la necrosis de las hojas, seguido por su abscisión, son unos de los síntomas más visibles del estrés por hipoxia radical, que, en casos extremos, puede significar la muerte de la planta en la medida que aumente el tiempo de exposición a este tipo de estrés. La disminución de la clorofila de plantas anegadas en plantas de tomate la observaron también Ezin et al. (2010) y Perez et al. (1999) y Aldana et al. (2014) en uchuva, mientras Ashraf (2012) afirma que la senescencia foliar prematura es una consecuencia de la inhibida tasa fotosintética originada por el cierre estomático bajo estas condiciones estresantes.

El entorchamiento o epinastia bajo condiciones de anaerobiosis o de inundación del suelo la describen Jordán y Casaretto (2006) en plantas de tomate como una mayor elongación de la porción adaxial superior del pecíolo respecto a la abaxial inferior. También Kuo y Chen (1980) observaron en plantas de tomate, anegadas 
durante $3 \mathrm{~d}$, un incremento de la curvatura de los peciolos foliares. Gracias a la epinastia, la planta puede disminuir la transpiración, protegiendo sus hojas contra el calor y el sol directo (Schaffer et al., 2006).

\section{CONCLUSIONES}

El efecto del anegamiento fue más severo en las variables altura del tallo, número de hojas y frutos cuando las plantas fueron anegadas durante 8 o 12 d y esta reducción se agravó hasta el día 40 de la evaluación.

La producción de biomasa seca fue reducida significativamente en las plantas, 8 y $12 \mathrm{~d}$ anegadas, sobre todo en frutos y raíces, indicando un efecto prejudicial sobre la función y el crecimien- to de las raíces, además de la fotosíntesis.

El tratamiento de sombreamiento solo no mostró un efecto significativo sobre el crecimiento de las plantas, indicando una cierta adaptación del tomate a esta situación, sin embargo, la combinación de los dos estreses (interacción 12 $\mathrm{d}$ anegamiento $\mathrm{x}$ sombrío) redujo más la acumulación de biomasa de la planta.

Como adaptaciones al estrés por anegamiento, las plantas mostraron amarillamiento, epinastia y abscisión de las hojas, probablemente para reducir la transpiración, además formaron hojas antociánicas con menos clorofila. El desarrollo de raíces adventicias, cerca del cuello de raíces, se ve como una medida de la planta de contrarrestar la falta de oxígeno en la zona subterránea.

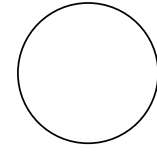

Aldana, F., P.N. García y G. Fischer. 2014. Effect of waterlogging stress on growth, development and symptomatology of cape gooseberry (Physalis peruviana L.) plants. Agron. Colomb. 32(2) (en imprenta).

Armstrong, W. y M.C. Drew. 2002. Root growth and metabolism under oxygen deficiency. pp. 729-761. En: Waisel, Y., A. Eshel y U. Kafkafi (eds.). Plant roots. The hidden half. 3th ed. Marcel Dekker, New York, NY.

Bradford, K.J. y S.F. Yang. 1981. Physiological responses of plants to waterlogging. HortScience 16, 25-30.

Bouzo, C. C. Freyre, E. Bouchet, J Favaro, R. Pilatti, J. Ramos y V. Dovis. 2008. Efecto del sombreo en cultivos de tomate (Lycopersicom esculentum Mill.) al aire libre. Facultad de Ciencias Agrarias, Universidad Nacional del Litoral, Santa Fe, Argentina.

Canovas, F. 1995. Manejo del cultivo sin suelo. pp. $227-$ 254. En: Nuez, F. (ed.). El cultivo del tomate. Ediciones Mundi-Prensa, Madrid.

Casierra-Posada, F. y N.E. Gómez. 2008. Crecimiento foliar y radical en plantas de fique (Furcraea castilla

\section{REFERENCIAS BIBLIOGRÁFICAS}

y F. macrophylla) bajo estrés por encharcamiento. Agron. Colomb. 26(3), 381-388.

Casierra-Posada, F. y Y.A. Vargas. 2007. Crecimiento y producción de fruta en cultivares de fresa (Fragaria sp.) afectados por encharcamiento. Rev. Colomb. Cienc. Hortic. 1(1), 21-32.

Corpoica, 2006. Cultivo de tomate bajo invernadero. Boletín Técnico 21. C.I. La Selva. Rionegro, Colombia.

Das, H.P. 2012. Agrometeorology in extreme events and natural disasters. BS Publikations, Hyderabad, India.

Ezin, V., R. de la Pena y A. Ahanchede. 2010. Flooding tolerance of tomato genotypes during vegetative and reproductive stages. Braz. J. Plant Physiol. 22(1), 131-142.

Fischer, G. y J.O. Orduz-Rodríguez. 2012. Ecofisiología en frutales. pp. 54-72. En: Fischer, G. (ed.). Manual para el cultivo de frutales en el trópico Produmedios, Bogotá.

Fischer, G. y C.P. Pérez. 2012. Efecto de la radiación solar en la calidad de los productos hortícolas. Me- 
morias del Congreso Internacional de Hortalizas en el Trópico (en CD). 28-30 Nov. 2012, Bogotá. Sociedad Colombiana de Ciencias Hortícolas, Bogotá.

Fischer, G., F. Ramírez y P.J. Almanza-Merchán. 2012. Inducción floral, floración y desarrollo del fruto. pp. 120-140. En: Fischer, G. (ed.). Manual para el cultivo de frutales en el trópico. Produmedios, Bogotá.

Heuvelink, E. y M. Dorais. 2005. Crop growth and yield. pp. 85-114. En: Heuvelink, E. (ed.). Tomatoes. CAB International, Wallingford, UK.

Iacona, C., M. Cirilli, A. Zega, E. Frioni, C. Silvestri y R. Muleo. 2013. A somaclonal myrobalan rootstock increases waterlogging tolerance to peach cultivar in controlled conditions. Sci. Hortic. 156, 1-8.

Jackson, M.B. y T.D. Colmer. 2005. Response and adaptations by plants to flooding stress. Ann. Bot. 96, 501-505.

Jiménez, J., L.P. Moreno y S. Magnitskiy. 2012. Respuesta de las plantas a estrés por inundación. Una revisión. Rev. Colomb. Cienc. Hortic. 6(1), 96-109.

Jordán, M. y J. Casaretto. 2006. Hormonas y reguladores del crecimiento: etileno, ácido abscísico, brasinoesteroides, poliaminas, ácido salicílico y ácido jasmónico. En: Squeo, F.A. y L. Cardemil (eds.). Fisiología vegetal. Ediciones Universidad de La Serena, La Serena, Chile.

Kozlowski, T.T. y S.G. Pallardy. 1997. Physiology of woody plants. Academic Press, San Diego, CA.

Koppitz, H. 2004. Effects of flooding on the amino acid and carbohydrate patterns of Phragmites australis. Limnologica 34, 37-47.

Kuo, C.G. y B.W. Chen. 1980. Physiological response of tomato cultuvars to flooding. J. Amer. Soc. Hort. Sci. 105(5), 751-755.

Lopez, O.R. y T.A. Kursar. 2003. Does flood tolerance explain tree distribution in seasonally flooded forests?. Oecologia 132, 193-204.

Lorbiecke, R. y M. Sauter. 1999. Adventitious root growth and cell-cycle induction in deepwater rice, Plant Physiol. 119, 21-29.

Mittler, R. 2002. Oxidative stress, antioxidants and stress tolerance. Trends Plant Sci. 7(9), 405-410.

Mittler, R. 2006. Abiotic stress, the field environment and stress combination. Trends Plant Sci. 11(1), 15-19.
Neuman, D.S. 1993. Shoot responses to root stress a resource gathering point of view. J. Arboricult. 19(2), 118-123.

Nicola, S., G. Tibaldi y E. Fontana. 2009. Tomato production systems and their application to the tropics. Acta Hort. 821, 27-33.

Parolin, P. 2009. Submerged in darkness: adaptations to prolonged submergence by woody species of the Amazonian floodplains. Ann. Bot. 103, 359-376.

Perez, O., J.M. Dell'Amica e I. Reynaldo. 1999. Performance of tomato seedlings under soil flooding. Cultivos Trop. 20(2), 41-44.

Rao, R. y Y. Li. 2003. Management of flooding effects on growth of vegetable and selected field crops. HortTechnol. 13(4), 610-616.

Reich, P. y M. Walters. 1992. Leaf life-span in relation to leaf, plant, and stand characteristics among diverse ecosystems. Ecol. Monographs 62(3), 365-392.

Rutto, K.L., F. Mizutani y K. Kadoya, K. 2001. Effect of root-zone flooding on micorrizal and non-mycorrhizal peach (Prunus persica Batsch) seedlings. Sci. Hortic. 94, 285-295.

Sauter, M. 2013. Root responses to flooding. Curr. Opinion Plant Biol. 16, 282-286.

Schaffer, B., F.S. Davies y J.H. Crane. 2006. Responses to tropical and subtropical fruit trees to flooding in calcareous soils. HortScience 41(3), 549-555.

Shiu, O.Y., J.H. Oetiker, W.K. Yip y S.F. Yang. 1998. The promoter of LE-ACS7, an early flooding-induced 1-aminocyclopropane-1-carboxylate synthase gene of the tomato, is tagged by a Sol3 transposon. Proc. Natl. Acad. Sci. USA 95, 10334-10339.

Ulrich, L. 1997. Physiological ecology of tropical plants. Springer, Berlín.

Vidoz, M.L., E. Loreti, A. Mensuali, A. Alpi y P. Perata. 2010. Hormonal interplay during adventitious root formation in flooded tomato plants. Plant J. $63,551-562$.

Wood, S., K. Sebastian y S. Scherr, S. 2000. Soil resource condition. En: Pilot analysis of global ecosystems: Agroecosystems. International Food Policy Research Institute and the World Resources Institute, Washington D.C.

Yin, D., S. Chen, F. Chen, Z. Guan y W. Fang. 2010. Morpho- anatomical and physiological responses of two Dendranthema species to waterlogging. Environ. Exp. Bot. 68, 122-130. 